\title{
Mixed Type Delusional Disorder
}

National Cancer Institute

\section{Source}

National Cancer Institute. Mixed Type Delusional Disorder. NCI Thesaurus. Code C94388.

A subtype of delusional disorder applied when no one delusional theme (such as

grandiose, jealous, persecutory) predominates. 